Hierarchical hexagonal boron nitride nanowall-diamond nanorod heterostructures with enhanced optoelectronic performance

Peer-reviewed author version

KAMATCHI JOTHIRAMALINGAM, Sankaran; Duc Quang Hoang; Korneychuk, Svetlana; Kunuku, Srinivasu; Thomas, Joseph Palathinkal; POBEDINSKAS, Paulius; DRIJKONINGEN, Sien; VAN BAEL, Marlies; D'HAEN, Jan; Verbeeck, Johan; Leou, Keh-Chyang; Leung, Kam Tong; Lin, I. -Nan \& HAENEN, Ken (2016) Hierarchical hexagonal boron nitride nanowall-diamond nanorod heterostructures with enhanced optoelectronic performance. In: RSC ADVANCES, 6(93), p. 90338-90346.

DOI: 10.1039/c6ra19596b

Handle: http://hdl.handle.net/1942/22767 


\section{Hierarchical hexagonal boron nitride nanowall-diamond nanorod heterostructures with enhanced optoelectronic performance}

Kamatchi Jothiramalingam Sankaran, ${ }^{\text {ab }}$ Duc Quang Hoang, ${ }^{\text {ab }}$ Svetlana Korneychuk, ${ }^{\mathrm{c}}$ Srinivasu Kunuku, ${ }^{\text {d Joseph Palathinkal Thomas, }}$, Paulius Pobedinskas, ${ }^{\text {ab }}$ Sien Drijkoningen, ${ }^{\text {ab }}$ Marlies K. Van Bael, ${ }^{\mathrm{ab}}$ Jan D'Haen, ${ }^{\mathrm{ab}}$ Johan Verbeeck, ${ }^{\mathrm{c}}$ Keh-Chyang Leou, ${ }^{\mathrm{d}}$ Kam Tong Leung, ${ }^{\mathrm{e}}$ I-Nan Lin, ${ }^{\mathrm{f}}$ and Ken Haenen*ab

A superior field electron emission (FEE) source made from a hierarchical heterostructure, where two-dimensional hexagonal boron nitride $(\mathrm{hBN})$ nanowalls coated on one-dimensional diamond nanorods (DNRs), is fabricated using a simple and scalable method. FEE characteristics of hBNDNR display a low turn-on field of $6.0 \mathrm{~V} / \mu \mathrm{m}$, a high field enhancement factor of 5870 and a high life-time stability of 435 min. Such an enhancement in the FEE properties of hBN-DNR derives from the distinctive materials combination, i.e., high aspect ratio of the heterostructure, good electron transport from the DNR to the $\mathrm{hBN}$ nanowalls and efficient field emission of electrons from the hBN nanowalls. The prospective application of these heterostructures is further evidenced by enhanced microplasma devices using hBN-DNR as cathode, in which the threshold voltage was lowered to $350 \mathrm{~V}$, affirming the role of hBN-DNR in the improvement of electron emission.

Keywords: hierarchical heterostructure, diamond nanords, hexagonal boron nitride nanowalls, electron field emission, microplasma illumination

\section{Introduction}

One dimensional (1D) hierarchical heterostructures consisting of two important functional materials have attracted much attention for developing potential applications in future nanoelectronic and optoelectronic devices due to their exceptional properties, which are different from bulk material properties. ${ }^{1-9}$ These heterostructures offer additional prospects for improving the functionality of 1D nanostructures which could lead to applications in nanoscale heterostructured electronic devices. ${ }^{10-12}$ Direct fabrication of the $1 \mathrm{D}$ heterostructures with 
controlled structural characteristics (comprising morphology, surface architectures, dimensionality, and crystal structures) signifies an important challenge in the field of nanoscience and nanotechnology. Recently, heterostructures, such as $\mathrm{ZnO}-\mathrm{Zn}_{3} \mathrm{P}_{2},{ }^{2} \mathrm{ZnS}-\mathrm{In},{ }^{13}$ $\mathrm{In}_{2} \mathrm{O}_{3}-\mathrm{Ga}_{2} \mathrm{O}_{3},{ }^{14} \mathrm{CdS}-\mathrm{CdSe},{ }^{15} \mathrm{ZnO}$-ultrananocrystalline diamond, ${ }^{16} \mathrm{ZnSe} / \mathrm{GeSe},{ }^{17} \mathrm{InAs}-\mathrm{InP},{ }^{18} \mathrm{Ag}$ $\mathrm{Ag}_{2} \mathrm{~S},{ }^{19} \mathrm{MoS}_{2}-\mathrm{TiO}_{2},{ }^{20}$ and $\mathrm{hBN}$-carbon nanotubes, ${ }^{21}$ have exhibited great potential in the field of photodetectors, energy storage devices, solar cells and field electron emitters.

High quality field electron emitters are anticipated for applications in a broad range of field emission based devices such as flat panel displays, high energy accelerators, electron microscopes, X-ray sources, vacuum microwave amplifiers, and cathode-ray tube monitors. Up to now, various field electron emission (FEE) cold cathode 1D nanostructured materials, for instance carbon nanotubes, $\mathrm{GaN}, \mathrm{Si}, \mathrm{SiC}, \mathrm{NiSi}, \mathrm{ZnO}, \mathrm{ZnS}, \mathrm{CdS}$, graphene, $\mathrm{Bi}_{2} \mathrm{Se}_{3}, \mathrm{SnO}_{2}$, and AlN nanostructures have been demonstrated as candidates for achieving enhanced FEE properties owing to their high aspect ratios. ${ }^{22-24}$ Besides the aspect ratio, the tops of the $1 \mathrm{D}$ nanostructured materials are not sharp enough for a very high local electrical field. ${ }^{25}$ In contrast, the aspect ratios of the 2-dimensional (2D) nanostructures are generally low, but the presence of a large number of sheet edges regularly exhibit many sharp tips, which can also lead to high local fields.

Motivated by the desire to achieve high performance FEE devices by combining the advantages of 1D and 2D nanostructured materials, herein, we fabricated a new architecture of field emitters by using hierarchical heterostructures of hBN nanowalls on diamond nanorods (hBN-DNR). The detailed morphological and structural features of the newly developed heterostructures are analyzed and discussed with respect to their excellent FEE performance in terms of low turn-on field, high field enhancement factor and high life-time stability. The promising FEE performances suggest a great potential of the hBN-DNR as a competitive candidate for future field emitters.

\section{Experimental methods}

2.1 Synthesis of hBN-DNR heterostructures. $n$-type silicon ( $\mathrm{Si}$ ) substrates of size $1 \mathrm{~cm}$ x $1 \mathrm{~cm}$ were cleaned with sulfuric acid/hydrogen peroxide and ammonia/hydrogen peroxide mixtures, 
respectively. The cleaned $\mathrm{Si}$ substrates were then seeded with a water-based state-of-the-art colloidal suspension of $5 \mathrm{~nm}$ detonation nanodiamonds. ${ }^{26}$ Second, the nanocrystalline diamond (NCD) film was grown on Si substrates by ASTeX 6500 series microwave plasma enhanced chemical vapor deposition (MPECVD) reactor for $4 \mathrm{~h}$. A gas mixture of $\mathrm{CH}_{4}, \mathrm{H}_{2}$ and $\mathrm{N}_{2}$ with flow rates of 18, 267 and $15 \mathrm{sccm}$, respectively $\left(\mathrm{CH}_{4} / \mathrm{H}_{2}=6 \%, \mathrm{~N}_{2} / \mathrm{H}_{2}=5 \%\right)$, was excited by 3000 $\mathrm{W}$ microwave power, and the total pressure in the chamber was maintained at 20 Torr. The Third, the grown NCD film was then immersed in a pseudo-stable suspension (nanodiamond (ND) particles (8 to $10 \mathrm{~nm}$ in diameter) and deionized water) and sonicated for $10 \mathrm{~min}$ to seed ND particles on the NCD film surface to serve as mask for reactive ion etching (RIE) etching process. The number density of ND particles on the NCD film depends on the suspension quality and time of sonication. After masking, the NCD film was then etched using the RIE process in $\mathrm{O}_{2}$ gas at a $\mathrm{RF}$ power of $200 \mathrm{~W}$ for $30 \mathrm{~min}$. The fabrication of nanostructures from diamond materials, which are extremely hard and chemically inert materials, is a very difficult task. Lots of effort exploring the possible techniques for fabricating the DNRs were made and eventually achieved a simple RIE process in $\mathrm{O}_{2}$ plasma to fabricate DNRs from NCD film using ND particles as mask. Finally, hBN nanowalls were synthesized on the DNRs by a home-built unbalanced 13.56 $\mathrm{MHz}$ radio frequency $(\mathrm{RF})$ sputtering technique. An optimal condition for fabrication of hBN nanowalls are gas mixture $\operatorname{Ar}(51 \%) / \mathrm{N}_{2}(44 \%) / \mathrm{H}_{2}(5 \%)$ and cathode power of $75 \mathrm{~W}$ with working pressure and target-to-substrate distance were $2.1 \times 10^{-2}$ mbar and $3 \mathrm{~cm}$, respectively. Herein, a 3 inch-diameter pyrolytic boron nitride ceramic with material purity and mass-density of $99.99 \%$ and $1.96 \times 10^{3} \mathrm{~kg} / \mathrm{m}^{3}$, respectively, was used as target. ${ }^{27,28}$ The time for the growth of hBN nanowalls on DNRs was $30 \mathrm{~min}$.

2.2 Morphological and structural characterization. The hBN-DNR heterostructures were characterized by confocal micro-Raman spectroscopy, Fourier transform infrared (FTIR) spectroscopy, X-ray photoelectron spectroscopy (XPS), scanning electron microscopy (SEM), high angle annular dark field scanning transmission electron microscopy (HAADF-STEM) and STEM-electron energy loss spectroscopy (EELS) using, respectively, a Horiba Jobin-Yuan T64000 spectrometer, FTIR NICOLET 8700 spectrometer, a Thermo-VG Scientific ESCALab 250 Microprobe (equipped with a monochromatic Al Ka X-ray source (1486.6 eV)), FEI Quanta 200 FEG microscope and a FEI Titan 'cubed' microscope operated at $300 \mathrm{kV}$ for HAADF- 
STEM-EELS. The convergence semi-angle $\alpha$ used was $22 \mathrm{mrad}$, the inner acceptance semi-angle $\beta$ for HAADF-STEM imaging was $22 \mathrm{mrad}$, the EELS collection angle used was also $22 \mathrm{mrad}$. The STEM specimens of these samples were prepared by the focused ion beam technique.

2.3 FEE measurements. FEE properties of the hBN-DNR heterostructures were measured using a tunable parallel plate set-up, in which the sample (hBN-DNR)-to-anode (Mo tip with a diameter of $3 \mathrm{~mm}$ ) distance was controlled using a micrometer. The schematic of our FEE measurement is shown in Fig. S1, ESI†. The current-voltage $(I-V)$ characteristics were measured using an electrometer (Keithley 2410) under pressure below $10^{-6}$ Torr.

2.4 Microplasma device measurements. The microplasma device was fabricated using indium tin oxide coated glass as the anode and hBN-DNR as the cathode. The schematic of our microplasma device measurement is shown in Fig. S2, ESI $\dagger$. The cathode-to-anode separation was fixed by a polytetrafluoroethylene spacer (1.0 $\mathrm{mm}$ in thickness), which includes a circular hole of about $3.0 \mathrm{~mm}$ in diameter as plasma cavity. The plasma was triggered using a pulse dc mode (a $20 \mathrm{~ms}$ square pulse and a $6 \mathrm{kHz}$ repetition rate) in Ar environment ( 2 Torr). The plasma current versus applied voltage behavior was measured using an electrometer (Keithley 237).

\section{Results and discussion}

The whole fabrication procedure for $\mathrm{hBN}$ nanowalls grown on DNRs, forming hierarchical hBNDNR heterostructures, is schematically presented in Fig. 1. The NCD film was first grown on $\mathrm{Si}$ substrates using an ASTeX 6500 series MPECVD system (Fig. 1a). A SEM image shown in the inset of Fig. 2a reveals that the NCD film contains a nano-grained microstructure with very smooth surface. The root-mean square roughness of the surface is about $10 \mathrm{~nm}$, and the thickness of the films is about $600 \mathrm{~nm}$. The surface of the NCD film was then masked using a pseudostable suspension containing ND particles in deionized water (Fig. 1b). The ND particles served as etching mask for fabricating vertically aligned DNRs (Fig. 1c). The NCD film was then etched using a RIE process in an $\mathrm{O}_{2}$ plasma. Fig. 2a shows the tilted SEM image of the vertically aligned DNRs with diameters of $\sim 40 \mathrm{~nm}$ and lengths of about $230 \mathrm{~nm}$. $\mathrm{hBN}$ nanowalls were then coated on the DNRs by a RF sputtering technique (Fig. 1d). ${ }^{27,28}$ As displayed in Fig. 2b, the whole surfaces of the DNRs are conformally covered with hBN nanowalls, which are of compact and curled morphology, thereby resulting in a nanoscale hierarchical heterostructure. The individual hierarchical hBN-DNR heterostructure has a much larger diameter than the pristine 
DNR. The SEM-energy dispersive X-ray (SEM-EDX) spectrum of the hBN-DNR shown in the inset of Fig. 2b revealed the presence of B, C, N, O and Si.

Fig. 2c displays the confocal micro-Raman spectrum of the hBN-DNR, which is deconvoluted using the multi-peak Lorentzian fitting method. Four prominent resonance peaks are observed in the spectrum. The broadened Raman peak at $\sim 1348 \mathrm{~cm}^{-1}$ is attributed to the Dband, which arises from disordered carbon, while the peak observed at $\sim 1558 \mathrm{~cm}^{-1}$, assigned as the G-band, is arising from the graphitic phase in the DNRs. ${ }^{29,30}$ The broad resonance peaks $v_{1^{-}}$ band $\left(1186 \mathrm{~cm}^{-1}\right)$ and $v_{3}$-band $\left(1526 \mathrm{~cm}^{-1}\right)$ correspond to the deformation modes of $\mathrm{CH}_{\mathrm{x}}$ bonds in the DNRs. ${ }^{31}$ The resonance peak at $1332 \mathrm{~cm}^{-1}$ (indicated by an arrow) corresponds to the $\mathrm{F}_{2 \mathrm{~g}}$ resonance mode of the $3 \mathrm{C}$ diamond lattice. A small peak corresponding to the $\mathrm{hBN}$ signal is barely observed at $1370 \mathrm{~cm}^{-1}, 32,33$ which is overlapped with the D band $\left(1348 \mathrm{~cm}^{-1}\right)$ of the DNRs. FTIR spectroscopy measurements were performed to examine the bonding characteristics of these hBN-DNR. The inset of Fig. 2c shows a sharp absorption peak at $783 \mathrm{~cm}^{-1}$ and a broad absorption band in the range of $1300-1500 \mathrm{~cm}^{-1}$, which were attributed to the $A_{2 u}(B-N-B$ bending vibration mode parallel to the $c$-axis) and $\mathrm{E}_{1 \mathrm{u}}$ (B-N stretching vibration mode perpendicular to the $c$-axis) modes of $\mathrm{hBN},{ }^{34-36}$ respectively. In addition, the peak at $1238 \mathrm{~cm}^{-1}$ can be consigned to the stretching vibration of B-C bonds. ${ }^{37,38}$ The absorption band centered at $1238 \mathrm{~cm}^{-1}$ can also be associated to the stretching vibration of $\mathrm{C}-\mathrm{N}$ bonds. ${ }^{38,39}$ Furthermore, the formation of $s p^{2} \mathrm{C}-\mathrm{N}$ bonds could contribute to the small absorption peak at $1564 \mathrm{~cm}^{-1}$, respectively, ${ }^{37,40}$ implying that there is some carbon species incorporated into the $\mathrm{hBN}$ nanowalls.

The chemical composition of the hBN-DNR was further analyzed using XPS. Fig. 3 shows a typical XPS survey, revealing that hBN-DNR are composed of B $(190 \mathrm{eV}), \mathrm{C}(285 \mathrm{eV}), \mathrm{N}(398$ $\mathrm{eV}$ ) and $\mathrm{O}(532 \mathrm{eV}) . \mathrm{B}, \mathrm{N}$ and $\mathrm{C}$ are the main ingredients in the hBN-DNR, whereas $\mathrm{O}$ is possibly due to physically adsorbed oxygen on the surface. To confirm the structure of hBN from XPS data, the B1s and N1s peaks in Fig. 3a are shown at a higher magnification in Fig. 3b and c, respectively. $\mathrm{hBN}$ shows bulk plasmon loss peaks at $\sim 23 \mathrm{eV}$ and $\sim 24 \mathrm{eV}$ away from the main B1s and N1s peaks. ${ }^{41}$ The $\pi$-plasmon loss peaks of the hBN-DNR are observed at a distance of $\sim 9 \mathrm{eV}$ from both B1s and N1s peaks, authenticating the $s p^{2}$ bonding and the hexagonal structure 
of $\mathrm{hBN}^{42}$ The major asymmetric C1s peak shown in Fig. 3d designates the existence of C-B $(285.2 \mathrm{eV})$ and $\mathrm{C}-\mathrm{N}(286.7 \mathrm{eV})$ bonds in the $\mathrm{hBN}-\mathrm{DNR}$ besides dominant $\mathrm{C}-\mathrm{C}$ bonds $(285.2 \mathrm{eV})$ from the DNRs. A contribution of C-O bonds at $289.4 \mathrm{eV}$ is attributed to oxygen contamination formed at the surface of the samples due to air exposure. The deconvoluted B1s XPS spectrum given in Fig. 3e mainly shows two sub-peaks at $190.6 \mathrm{eV}$ and $191.2 \mathrm{eV}$. While the binding energy of $191.2 \mathrm{eV}$ corresponds to B-N bonds, a lower binding energy of $190.6 \mathrm{eV}$ for B 1s suggests a contribution from the bonding configurations of B and C. ${ }^{43,44}$ The N1s XPS spectrum (Fig. 3f) further confirms the bonding configuration between $\mathrm{N}$ and $\mathrm{C}$ and $\mathrm{B}$ and $\mathrm{N}$, respectively, in the hBN-DNR.

Further details of the microstructure of hBN-DNR were disclosed by the HAADF-STEM and high resolution STEM (HR-STEM) observation. Fig. 4a shows a typical cross-sectional HAADF-STEM micrograph of the heterostructures, in which the hBN-DNR and the NCD film regions are clearly marked. Fig. 4a displays that the DNRs were fully covered with hBN nanowalls. Fig. 4b shows a HR-STEM image obtained from a region at the hBN-diamond interface (region "A" designated in Fig. 4a). It can be seen that the hBN nanowalls grow directly on the diamond surface, without the formation of any precursor layers like amorphous $\mathrm{BN}(\mathrm{aBN})$ or turbostratic $\mathrm{BN}(\mathrm{tBN})$ prior to its nucleation. Highly ordered lattice fringes of hBN nanowalls can be observed, indicating that the hBN nanowalls are well crystallized. In addition, a Fourier transformed (FT) pattern (inset of Fig. 4b) corresponding to the $\mathrm{hBN}$ region illustrates the existence of the hBN phase. A higher magnification HR-STEM image of region " $\mathrm{B}$ " in Fig. 4b is presented in Fig. 4c, which reflects the crystalline nature of diamond, again confirmed by the FT image (inset of Fig. 4c). It is to be noted that the deposition of hBN nanowalls on Si first yields an interlayer of aBN followed by tBN phases. ${ }^{45}$ In this work, hBN nanowalls grow directly on DNRs without the formation of $\mathrm{aBN}$ and $\mathrm{tBN}$ phases as interfacial layer.

Closer inspection by high magnification HAADF-STEM of a single hBN-DNR heterostructure in Fig. 4d shows a collection of sharp edged hBN nanowalls which are spiked from the outer surface of the DNR. To illustrate more clearly the elemental distribution of the species, spatially resolved STEM- EELS mapping was performed. In the experiment, Fig. 4d was scanned using a fine probe, collecting a core-loss EELS spectrum containing the B-K, C-K and $\mathrm{N}-\mathrm{K}$ edges in each point. By integrating the intensity under the $\mathrm{B}, \mathrm{C}$ and $\mathrm{N}$ edges, elemental 
maps were generated and composed. Fig. 4e shows a micrograph composed of the STEM-EELS mapping with diamond (green) and hBN (pink) for the same region depicted in Fig. 4d. In Fig. 4f, two summed selective area EELS spectra from the diamond and the hBN regions in Fig. $4 \mathrm{e}$ are plotted. The carbon K-edge spectrum acquired from the diamond region is typical of $s p^{3}$ carbon, with a strong $\sigma^{*}$ contribution at $292 \mathrm{eV}$ and deep valley in $302 \mathrm{eV}$. ${ }^{46,47}$ The EELS spectrum corresponding to the $\mathrm{hBN}$ region of Fig. 4e exhibits two distinct edges; the boron-K $188 \mathrm{eV}$ and the nitrogen-K at $401 \mathrm{eV} \cdot{ }^{48-50}$ The fine structure of the $\mathrm{B}-\mathrm{K}$ and $\mathrm{N}-\mathrm{K}$ edges are typical of the $s p^{2}$-coordinated layered $\mathrm{BN}$, indicating that the obtained nanowalls are $\mathrm{hBN}$ with hexagonal layered structure. In addition to the core-loss K-edges of $\mathrm{B}$ and $\mathrm{N}$, the residual presence of carbon is also detected through the presence of a core-loss carbon-K edge at $285 \mathrm{eV}$ ( $\pi^{*}$ band). The fine structure of the carbon $\mathrm{K}$-edge is typical of amorphous carbon $(a-C)$, confirming that an $a-C$ phase is present in the hBN-DNR heterostructures. It is again confirming from the STEM-EELS map shown in Fig. $4 \mathrm{~g}$ that $a-C$ phase (red) is present in the DNR structures (region I of Fig. $4 \mathrm{~g}$ ). The $a-C$ phase may be present in the grain boundaries of the DNRs. ${ }^{51}$ In addition, $a-C$ has been incorporated into the $\mathrm{hBN}$ region (region II of Fig. $4 \mathrm{~g}$ ). These STEM-EELS results together with the elemental maps indicate the existence of B-N, B-C, and C-N bonds within the hBN-DNR heterostructures, confirming the FTIR and XPS data (cf. inset of Fig. 2c and Fig. 3). Leung et al. also examined the diffusion of $\mathrm{C}$ in the interface during the growth of cubic $\mathrm{BN}$ on amorphous tetrahedral carbon interlayers. ${ }^{52}$ The presence of $\mathrm{C}$ in the interface region is possibly induced by carbon incorporation and dynamic recoil ion mixing in an early stage of boron nitride deposition. This incorporated carbon region then relates to a C-B-N gradient layer, which may contribute to the interfacial stress relaxation. On the basis of FTIR, XPS and STEM-EELS observations, it is obvious that the hBN nanowalls nucleated and grew directly on the DNR surface, doing so inhibiting the formation of $\mathrm{aBN}$ and $\mathrm{tBN}$ phases in the interface. In addition, $\mathrm{C}$ species were incorporated in the $\mathrm{hBN}$ nanowalls.

In order to study the performance of the hBN-DNR heterostructures as a field emitter, FEE characteristics were measured in a high vacuum of $10^{-6}$ Torr. For comparison, hBN nanowalls were also fabricated directly on the $\mathrm{Si}$ substrate and were designated as hBN-Si. The relations between the FEE current density and the electric field ( $J_{\mathrm{e}}-E$ curves) of hBN-Si and hBN-DNR heterostructures are both given in Fig. 5a and were modeled using the Fowler-Nordheim (F-N) 
formula. ${ }^{53}$ Here, $J_{\mathrm{e}}$ is obtained by dividing the total emission current by the sample area and $E$ is obtained by dividing the voltage by the spacing between the anode and the cathode. The FEE properties of these samples were characterized by their turn-on fields $\left(E_{0}\right)$. The $E_{0}$ for inducing the FEE process was determined from the intersection of two lines extrapolated from the lowfield and high-field segments of the F-N plots, which were plotted as $\ln J_{\mathrm{e}} / E^{2}$ versus $1 / E$ curves (inset of Fig. 5a). The FEE process of the hBN-DNR can be turned on at a considerably lower field of $\left(E_{0}\right)_{\mathrm{hBN}-\mathrm{DNR}}=6.0 \mathrm{~V} \mu \mathrm{m}^{-1}$, attaining a higher FEE current density of $\left(J_{\mathrm{e}}\right)_{\mathrm{hBN}-\mathrm{DNR}}=4.1 \mathrm{~mA}$ $\mathrm{cm}^{-2}$ at $E=14.0 \mathrm{~V} \mu \mathrm{m}^{-1}$ (curve II, Fig. 5a). In contrast, we observed markedly inferior FEE properties for hBN-Si with a $\left(E_{0}\right)_{\mathrm{hBN}-\mathrm{Si}}$ value of $40.2 \mathrm{~V} \mu \mathrm{m}^{-1}$ and a low FEE current density of

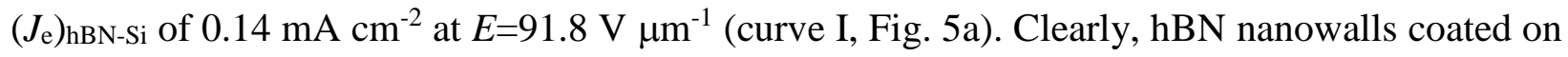
DNRs effectively promoted the field emission capability of the heterostructures. It is worth noting that the $E_{0}$ value of $\mathrm{hBN}-\mathrm{DNR}$ is comparable to the $E_{0}$ values of other heterostructures ${ }^{14,15,54-57}$ reported in literature, as summarized in Table 1.

According to the F-N model, ${ }^{53}$ the FEE is a quantum phenomenon where electrons are emitted from a material's surface into vacuum by tunneling through a potential barrier under the influence of a high electric field. The relationship between $J_{\mathrm{e}}$ and $E$ can be depicted as, $J_{\mathrm{e}}=$ $\left(A \beta^{2} E^{2} / \varphi\right) \exp \left(-B \varphi^{3 / 2} / \beta E\right)$, where $A$ and $\mathrm{B}$ are constants with values $1.54 \times 10^{-6} \mathrm{~A} \mathrm{eV} \mathrm{V}^{-2}$ and $6.83 \times 10^{9} \mathrm{eV}^{-3 / 2} \mathrm{~V} \mathrm{~m}^{-1}, \beta$ is the field enhancement factor and $\varphi$ is the work function of the emitting materials (the work functions are $5.0 \mathrm{eV}$ for diamond ${ }^{58}$ and $6.0 \mathrm{eV}$ for $\mathrm{hBN}^{59}$ ), respectively. We have estimated the $\beta$ from the slope of the F-N plot (straight line behavior in the low-field region, inset of Fig. 5a, which is mathematically expressed as, $\beta=\left[-6.8 \times 10^{3} \varphi\right.$ ${ }^{3 / 2} \mathrm{~J} / m$, where, $m$ is the slope of the F-N plot. Thus from the inset of Fig. $5 \mathrm{a}, \beta$ values for hBN-Si and hBN-DNR heterostructures were calculated to be 425 and 5870 (curves I and II, inset of Fig. $5 a)$. The $\beta$ value of hBN-DNR is higher than previously reported values of other heterostructures such as, $\mathrm{In}_{2} \mathrm{O}_{3}-\mathrm{Ga}_{2} \mathrm{O}_{3},{ }^{14} \mathrm{CdS}-\mathrm{CdSe},{ }^{15} \mathrm{~W}-\mathrm{WO}_{2.72},{ }^{54} \mathrm{LaNiO}_{3}-\mathrm{ZnO},{ }^{55} \mathrm{ZnO}-\mathrm{WO}{ }^{56}$ and $\mathrm{ZnS}$ tetrapod ${ }^{57}$ heterostructures (see Table 1). Generally, electrons transport along the nanorods; if there are sharp geometric protrusions on the outer surface of the nanorods, electrons can also emit from these protrusive regions in which there exists a higher $\beta$. The surface of each DNR is encased with hBN nanowalls, of which the nanowalls have a smaller curvature radius than that of the DNR and they become the prominent emission sites. Additionally, the well-aligned shape 
and suitable aspect ratio of DNR effectively decrease the screening effect, ${ }^{60}$ resulting in a high $\beta$ in this experiment.

For vacuum microelectronic device applications, FEE current stability is an important parameter. The FEE life-time stability measurements were evaluated by measuring the $J_{\mathrm{e}}$ as a function of time for these heterostructures. Fig. 5b shows that the emission current variations corresponding to $J_{\mathrm{e}}$ of $1.56 \mathrm{~mA} \mathrm{~cm} \mathrm{~cm}^{-2}$ recorded over a period of $435 \mathrm{~min}$ for $\mathrm{hBN}-\mathrm{DNR}$ at a working field of $13.0 \mathrm{~V} \mathrm{~m}^{-1}$. No significant current degradation was observed during the 435 min testing time. However, the hBN-Si (inset of Fig. 5b) shows the emission current variations recorded only a period of $28 \mathrm{~min}$ at a working field of $85.0 \mathrm{~V} \mu \mathrm{m}^{-1}$ corresponding to $J_{\mathrm{e}}$ of $0.1 \mathrm{~mA}$ $\mathrm{cm}^{-2}$. Such a long FEE life-time stability of hBN-DNR assures the practical application in field emitters.

The improved FEE behavior of the hBN-DNR can be explained as follows: first, the $a-C$ phase in the grain boundaries of DNR conducts the electrons efficiently to the hBN-DNR interface. Second, the direct growth of hBN nanowalls on the DNR surface lowers the resistivity of the interfacial layer and therefore the electrons can be transferred readily from DNR across the interfacial layer to the $\mathrm{hBN}$ nanowalls. Finally, the incorporation of $\mathrm{C}$ in the $\mathrm{hBN}$ nanowalls provides efficient electron transport paths for the emitted electrons to reach the tip of the nanowalls from which they escape into vacuum without any difficulty as the hBN surfaces are negative electron affinity in nature ${ }^{61}$ that reduces the $E_{0}$ value by lowering the barrier for the emitting electrons and thus enhances the FEE $J_{\mathrm{e}}$. Moreover, the vertically aligned hBN-DNR facing the anode could be considered as an additional reason for improvement of the FEE properties of $\mathrm{hBN}-\mathrm{DNR}$.

To appraise the robustness of the hBN-DNR, these heterostructures were utilized as cathodes for microplasma devices because the cathode in these devices experienced the continuous bombardment of energetic Ar ions, which is considered as the harshest environment in device applications. Fig. 6a displays a series of photographs of the microplasma devices, which were triggered by a pulsed direct current signal with increasing applied voltage at a pressure of 2 Torr. These micrographs show that the cathodic device using the hBN-DNR (image series II, Fig. 6a) performs much better than those using the $\mathrm{hBN}-\mathrm{Si}$ as cathode (image series I, Fig. 6a). The intensity of the plasma increases monotonically with the applied voltage. The 
microplasma behavior can be better illustrated by measuring the voltage dependence of plasma current density $\left(J_{\mathrm{pl}}-\mathrm{V}\right.$ curves), which are shown in Fig. $6 \mathrm{~b}$. The Ar-microplasma of the hBNDNR can be triggered by a voltage of as low as $350 \mathrm{~V}$, which corresponds to an applied field of $0.35 \mathrm{~V} \mathrm{\mu m}^{-1}$ (curve II, Fig. 6b). In contrast, the hBN-Si based microplasma device needs higher voltage, around $450 \mathrm{~V}$, which corresponds to an applied field of $0.45 \mathrm{~V} \mathrm{\mu m}^{-1}$, to trigger the plasma (curve I, Fig. 6b). The threshold field $\left(E_{\text {th }}\right)$ for hBN-Si based microplasma devices is comparatively larger than the $E_{\text {th }}$ value for the hBN-DNR based device. The plasma current density $\left(J_{\mathrm{pl}}\right)$ of the hBN-DNR based device reached $3.6 \mathrm{~mA} \mathrm{~cm}^{-2}$ (curve II in Fig. $6 \mathrm{~b}$ ) and the $J_{\mathrm{pl}}$ value of the $\mathrm{hBN}-\mathrm{Si}$ cathodic device can reach around $1.04 \mathrm{~mA} \mathrm{~cm}{ }^{-2}$ at an applied voltage of 540 $\mathrm{V}$, which corresponds to an applied field of $0.54 \mathrm{~V} / \mu \mathrm{m}$ (curve I, Fig. 6b).

The other eminent feature of using hBN-DNR as a cathode in microplasma devices is that it increased distinctly the life-time of the devices. The plasma intensity of the hBN-DNR based microplasma devices continues stable over $139 \mathrm{~min}$ (at $J_{\mathrm{pl}}$ of $1.95 \mathrm{~mA} \mathrm{~cm}^{-2}$ ), displaying the high stability of the hBN-DNR based microplasma devices (curves II, inset of Fig. 6b). In contrast, the $J_{\mathrm{pl}}$ value of $0.53 \mathrm{~mA} \mathrm{~cm}{ }^{-2}$ decreased fast after $29 \mathrm{~min}$ of plasma ignition for the $\mathrm{hBN}-\mathrm{Si}$ based microplasma devices (curve I, inset of Fig. 6b). From these results we concluded that the utilization of hBN-DNR as a cathode improved noticeably the robustness of the microplasma devices. It is to be noted that the cathode material experiences continuous bombardment by Arions with high kinetic energies $(400 \mathrm{eV})$ in the microplasma device, which is conceived as the harshest environment in the device applications. Presumably, the better plasma illumination performance of the microplasma devices based on the hBN-DNR, as associated with that of $\mathrm{hBN}$-Si based ones, is closely interrelated with the enhanced FEE properties of the hBN-DNR.

\section{Conclusions}

In summary, hierarchical hBN-DNR were successfully fabricated via a combination process of chemical vapor deposition synthesis for NCD film, RIE process for fabricating DNRs and the RF sputtering synthesis for hBN nanowalls. Covering 1D DNRs with 2D hBN nanowalls is an effective approach to utilize the advantages of both nanostructured materials in field emission device applications. FEE measurements of the material show a low $E_{0}$ value of $6.0 \mathrm{~V} \mathrm{\mu m}^{-1}$, a high $\beta$ value of 5870 , and high life-time stability of $435 \min$ (under $J_{\mathrm{e}}=1.56 \mathrm{~mA} \mathrm{~cm}{ }^{-2}$ ). The excellent FEE performance is attributed to the specific crystallographic feature of hBN 
nanowalls and DNR. The relatively large aspect ratio of the DNR and the sharp layer edges in the hBN nanowalls jointly contribute to the field enhancement. A large number of layer edges in the hBN nanowalls function as the emission sites. These results suggest that the new hBN-DNR may have a high promise for novel field-emitting and microplasma devices.

\section{Acknowledgements}

The authors like to thank the financial support of the Research Foundation Flanders (FWO) via Research Project G.0456.12 and the Methusalem "NANO" network. K J Sankaran, and P Pobedinskas are Postdoctoral Fellows of the Research Foundation-Flanders (FWO).

\section{Notes and references}

${ }^{a}$ Institute for Materials Research (IMO), Hasselt University, 3590 Diepenbeek, Belgium.

${ }^{b} I M O M E C$, IMEC vzw, 3590 Diepenbeek, Belgium.

Email: sankaran.kamatchi@uhasselt.be, ken.haenen@uhasselt.be.

${ }^{c}$ Electron Microscopy for Materials Science (EMAT), University of Antwerp, 2020 Antwerp, Belgium.

${ }^{d}$ Department of Engineering and System Science, National Tsing Hua University, 30013 Hsinchu, Taiwan.

${ }^{e}$ WATLab and Department of Chemistry, University of Waterloo, Waterloo, N2L3G1 Ontario, Canada.

${ }^{f}$ Department of Physics, Tamkang University, 251 Tamsui, Taiwan.

†Electronic supplementary information (ESI) available. See DOI:

1 L. J. Lauhon, M. S. Gudiksen, D. Wang and C. M. Lieber, Nature, 2002, 420, 57-61.

2 R. S. Yang, Y. L. Chueh, J. R. Morber, R. Snyder, L. J. Chou and Z. L. Wang, Nano Lett.,2007, 7, 269-275.

3 Y. L. Chueh, L. J. Chou and Z. L. Wang, Angew. Chem., Int. Ed., 2006, 45, 7773-7778. 
4 G. Shen, Y. Bando, Y. Gao and D. Golberg, J. Phys. Chem. B, 2006, 110, 1423-1427.

5 M. S. Gudiksen, L. J. Lauhon, J. Wang, D. C. Smith and C. M. Lieber, Nature, 2002, 415, $617-620$.

6 Y. Xia, P. Yang, Y. Sun, Y. Wu, B. Mayers, B. Gates, Y. Yin, F. Kim and H. Yan, Adv. Mater., 2003, 15, 353-389.

7 R. Tenne, Nat. Nanotechnol., 2006, 1, 103-111.

8 Y. Li, G. W. Meng, L. D. Zhang and F. Phillipp, Appl. Phys. Lett., 2000, 76, 2011-2013.

9 J. Q. Hu, Y. Bando, J. H. Zhan and D. Golberg, Adv. Mater., 2005, 17, 1964-1969.

10 G. Shen, D. Chen, Y. Bando, D. Golberg, J. Mater. Sci. Technol., 24, 2008, 541-549.

11 M. S. Gudiksen, L. J. Lauhon, J. Wang, D. C. Smith and C. M. Lieber, Nature, 2002, 415, $617-620$.

12 M. A. Verheijen, G. Immink, T. De Smet, M. T.Borgstrom and E. P. A.M.Bakkers, J. Am. Chem. Soc., 2006, 128, 1353-1359.

13 U. K. Gautam, X. Fang, Y. Bando, J. Zhan and D. Golberg, ACS Nano, 2008, 2, 1015-1021.

14 J. Lin, Y. Huang, Y. Bando, C. Tang, C. Li and D. Golberg, ACS Nano, 2010, 4, 2452-2458.

15 G. Li, Y. Jiang, Y. Zhang, X. Lan, T. Zhai and G. C. Yi, J. Mater. Chem. C., 2014, 2, 8252-8258.

16 K. J. Sankaran, M. Afsal, S. C. Lou, H. C. Chen, C. Chen, C. Y. Lee, L. J. Chen and N. H. Tai, Small, 2014, 10, 179-185.

17 Q. Xie, C. Wang, X. Xu, J. Liu and J. Zhang, Jpn. J. Appl. Phys., 2010, 49, 025001.

18 X. Jiang, Q. Xiong, S. Nam, F. Qian, Y. Li and C. M. Lieber, Nano Lett., 2007, 7, 3215-3218.

19 J. Xiong, C. Han, W. Li, Q. Sun, J. Chen, S. Chou, Z. Li and S. Dou, CrystEngComm, 2016, 18, 930-937.

20 J. Yang, J. Liang, G. Zhang, J. Li, H. Liu and Z. Shen, Vacuum, 2016, 123, 17-32.

21 X. Yang, Z. Li, F. He, M. Liu, B. Bai, W. Liu, Z. Qiu, H. Zhou, C. Li and Q. Dai, Small, 2015, 11, 3710-3716.

22 X. Fang, Y. Bando, U. K. Gautam, C. Ye and D. Golberg, J. Mater. Chem., 2008, 18, $509-522$. 
23 H. Huang, Y. Li, Q. Li, B. Li. Z. Song, W. Huang. C. Zhao, H. Zhang, S. Wen, D. Carroll and G. Fang, Nanoscale, 2014, 6, 8306-8310

24 H. Huang, C. K. Lin, M. S. Tse, J. Guo and O. K. Tan, Nanoscale, 2012, 4, 1491-1496.

25 J. Xiao, X. Zhang and G. Zhang, Nanotechnology, 2008, 19, 295706.

26 O. A. Williams, O. Douheret, M. Daenen, K. Haenen, E. Osawa and M. Takahashi, Chem. Phys. Lett., 2007, 445, 255-258.

27 B. BenMoussa, J. D’Haen, C. Borschel, J. Barjon, A. Soltani, V. Mortet, C. Ronning, M. D’Olieslaeger, H.-G. Boyen and K. Haenen, J. Phys. D: Appl. Phys., 2012, 45, 135302.

28 D. Q. Hoang, P. Pobedinskas, S. S. Nicley, S. Turner, S. D. Janssens, J. Verbeeck, M. K. Van Bael, J. D’Haen and K. Haenen, accepted for publication in Cryst. Growth Des., 2016, 16, 3699-3708.

29 A. C. Ferrari and J. Robertson, Phys. Rev. B, 2001, 63, 121405.

30 J. Michler, Y. Von Kaenel, J. Stiegler and E. Blank, J. Appl. Phys., 1998, 83, 187-197.

31 V. Mortet, L. Zhang, M. Eckert, J. D’Haen, A. Soltani, M. Moreau, D. Troadec, E. Neyts, J. C. D. Jaeger, J. Verbeeck, A. Bogaerts, G. V. Tendeloo, K. Haenen and P. Wagner, Phys. Status Solidi A, 2012, 209, 1675-1682.

32 R. Geick, C. H. Perry and G. Rupprecht, Phys. Rev., 1966, 146, 543-547.

33 J. Wu, W. Q. Han, W. Walukiewicz, J. W. Ager, W. Shan, E. E. Haller and A. Zettl, Nano Lett., 2004, 4, 647-650.

34 J. Yu and S. Matsumoto, Diamond Relat. Mater., 2004, 13, 1704-1708.

35 Z. G. Chen, J. Zou, G. Liu, F. Li, Y. Wang, L. Z. Wang, X. L. Yuan, T. Sekiguchi, H. M. Cheng and G. Q. Lu, ACS Nano, 2008, 2, 2183-2191.

36 E. Borowiak-Palen, T. Pichler, G. G. Fuentes, B. Bendjemil, X. Liu, A. Graff, G. Behr, R. J. Kalenczuk, M. Knupfer and J. Fink, Chem. Commun., 2003, 82-83.

37 Y. Wada, Y. K. Yap, M. Yoshimura, Y. Mori and T. Sasaki, Diamond Relat. Mater., 2000, 9, $620-624$.

38 X. M. Wu, X. M. Yang, L. J. Zhuge and F. Zhou, Appl. Surf. Sci., 2009, 255, 4279-4282.

39 T. Sugiyama, T. Tai and T. Sugino, Appl. Phys. Lett., 2002, 80, 4214-4216. 
40 H. D. Li, J. Lu, P. W. Zhu, X. Y. Lu and Y. G. Li, Appl. Surf. Sci., 2011, 257, 4963-4967.

41 A. Pakdel, X. Wang, C. Zhi, Y. Bando, K. Watanabe, T. Sekiguchi, T. Nakayama and D. Golberg, J. Mater. Chem., 2012, 22, 4818-4824.

42 J. H. Boo, S. B. Lee, K. S. Yu, Y. Kim, Y. S. Kim and J. T. Park and J. Korean, Phys. Soc., 1999, 34, S532-S537.

43 X. Liu, X. Jia, Z. Zhang, M. Zhao, W. Guo, G. Huang and H. A. Ma, Cryst. Growth Des., 2011, 11, 1006.

44 L. Ci, L. Song, C. Jin, D. Jariwala, D. Wu, Y. Li, A. Srivastava, Z. F. Wang, K. Storr, L. Balicas, F. Liu and P. M. Ajayan, Nat. Mater. 2010, 9, 430-435.

45 K. J. Sankaran, H. D. Quang, S. Kunuku, S. Korneychuk, S. Turner, P. Pobedinskas, S. Drijkoningen, M. K. Van Bael, J. D’Haen, J. Verbeeck, K. C. Leou, I. N. Lin and K. Haenen, Scientific Reports, 2016, 6, 29444.

46 S. S. Chen, H. C. Chen, W. C. Wang, C. Y. Lee, I. N. Lin, J. Guo and C. L. Chang, J. Appl. Phys. 2013, 113, 113704.

47 D. Zhou, T. G. McCauley, L. C. Qin, A. R. Krauss and D. M. Gruen, J. Appl. Phys., 1998, 83, $540-543$.

48 A. Loiseau, F. Willaime, N. Demoncy, G. Hug and H. Pascard, Phys. Rev. Lett., 1996, 76, 4737-4740.

49 M. Terrones, W. K. Hsu, H. Terrones, J. P. Zhang, S. Ramos, J. P. Hare, R. Castillo, K. Prassides, A. K. Cheetham, H. W. Kroto and D. R. M. Walton, Chem. Phys. Lett., 1996, 259, $568-573$.

50 W. Q. Han, Y. Bando, K. Kurashima and T. Sato, Appl. Phys. Lett., 1998, 73, 3085-3087.

51 K. J. Sankaran, K. Srinivasu, H. C. Chen, C. L. Dong, K. C. Leou, C. Y. Lee, N. H. Tai and I. N. Lin, J. Appl. Phys., 2013, 114, 054304.

52 K. M. Leung, C. Y. Chan, Y. M. Chong, Y. Yao, K. L. Ma, I. Bello, W. J. Zhang and S. T. Lee, J. Phys. Chem. B, 2005, 109, 16272-16277.

53 R. H. Fowler and L. Nordheim, Proc. R. Soc. London, Ser. A, 1928, 119, 173-181.

54 X. Liu, M. Song, S. Wang and Y. He, Physica E, 2013, 53,260-265. 
55 T. H. Yang, Y. W. Harn, K. C. Chiu, C. L. Fan and J. M. Wu, J. Mater. Chem., 2012, 22, 17071-17078.

56 H. Kim, S. Jeon, M. Lee, J. Lee and K. Yong, J. Mater. Chem., 2011, 21, 13458-13463

57 Z. G. Chen, J. Zou, G. Liu, X. Yao, F. Li, X. L. Yuan, T. Sekiguchi, G. Q. Lu and H. M. Cheng, Adv. Funct. Mater., 2008, 18, 3063-3069.

58 J. Liu, V. V. Zhirnov, A. F. Myers, G. J. Wojak, W. B. Choi, J. J. Hren, S. D. Wolter, M. T. McClure, B. R. Stoner and J. T. Glass, J. Vac. Sci. Technol. B, 1995, 13, 422-426.

59 J. Cumings and A. Zettl, Solid State Communications, 2004, 129, 661-664.

60 W. C. Chang, C. H. Kuo, C. C. Juan, P. J. Lee, Y. L Chueh and S. J. Lin, Nanoscale Res. Lett., 2012, 7, 684.

61 K. P. Loh, I. Sakaguchi, M. N. Gamo, S. Tagawa, T. Sugino and T. Ando, Appl. Phys. Lett., 1999, 74, 28-30. 
Table 1. Key field electron emission performance parameters of hBN-DNR heterostructures compared to other heterostructures reported in literature.

\begin{tabular}{|c|c|c|}
\hline Heterostructures & $\begin{array}{c}\text { Turn-on field } \\
E_{0}\left(\mathrm{~V} \mu \mathrm{m}^{-1}\right)\end{array}$ & $\begin{array}{c}\text { Field enhancement } \\
\text { factor }(\beta)\end{array}$ \\
\hline $\mathrm{In}_{2} \mathrm{O}_{3}-\mathrm{Ga}_{2} \mathrm{O}_{3}$ heterostructures ${ }^{14}$ & 6.45 & 4002 \\
\hline CdS-CdSe heterostructures ${ }^{15}$ & 9.0 & 550 \\
\hline $\mathrm{W}-\mathrm{WO}_{2.72}$ heterostructures $^{54}$ & 7.1 & 684 \\
\hline $\mathrm{LaNiO}_{3}-\mathrm{ZnO}$ nanorod arrays ${ }^{55}$ & 8.6 & 673 \\
\hline ZnO-WOx hierarchical nanowires ${ }^{56}$ & 3.6 & 2490 \\
\hline ZnS tetrapod tree-like heterostructures ${ }^{57}$ & 2.66 & 2600 \\
\hline hBN-DNR heterostructures ${ }^{\text {Present study }}$ & 6.0 & 5870 \\
\hline
\end{tabular}




\section{Figure captions}

Fig. 1. Schematics of the fabrication process of hBN-DNR heterostructures: (a) growth of NCD film on Si substrates; (b) masking ND particles on NCD films; (c) reactive ion etching for forming DNRs and (d) growth of hBN nanowalls on DNRs.

Fig. 2. (a) Tilted view SEM image of bare DNRs (inset shows the SEM morphology of NCD film). (b) tilted view SEM image for hBN-DNR with EDX spectrum shown as inset. (c) microRaman spectrum of hBN-DNR with FTIR spectrum shown as inset.

Fig. 3. (a) XPS survey spectrum of hBN-DNR, (b) the B1s and (c) the N1s peaks at a higher magnification, (d)-(f) deconvolutions of C1s, B1s and N1s edges of hBN-DNR heterostructures, respectively.

Fig. 4. (a) Typical cross-sectional HAADF-STEM image of the hBN-DNR. (b) HR-STEM image of a single hBN-DNR corresponding to region " $\mathrm{A}$ " of (a), with the FT patterns displayed in the inset of (b), disclosing the hBN phase. (c) Higher magnification HR-STEM image corresponding to the region " $\mathrm{B}$ " of (b), revealing the diamond structure and the FT pattern shown as in the inset of (c) evidences the crystalline nature of the diamond. (d) Typical HAADF-STEM micrograph of the hBN-DNR together with (e) composed EELS elemental mapping for hBN (pink) and DNR (green). (f) Summed EELS core-loss spectra taken from the diamond and hBN regions in the maps in (e). (g) EELS mapping of (d) disclosing the presence of $a-C$ in hBN-DNR heterostructures.

Fig. 5. (a) The field electron emission properties ( $\mathrm{J}_{\mathrm{e}}-E$ curves $)$ with $\mathrm{F}-\mathrm{N}$ plots as inset for I. hBN$\mathrm{Si}$ and II. hBN-DNR and (b) the current density versus time curves of hBN-DNR. The inset in (b) shows the current density versus time curve of $\mathrm{hBN}-\mathrm{Si}$.

Fig. 6. (a) The microplasma illumination images and (b) plasma current density versus applied voltage characteristics with the inset showing the plasma illumination stability, the life-time, of the microplasma devices, which were fabricated using I. hBN-Si and II. hBN-DNR as cathode materials. 


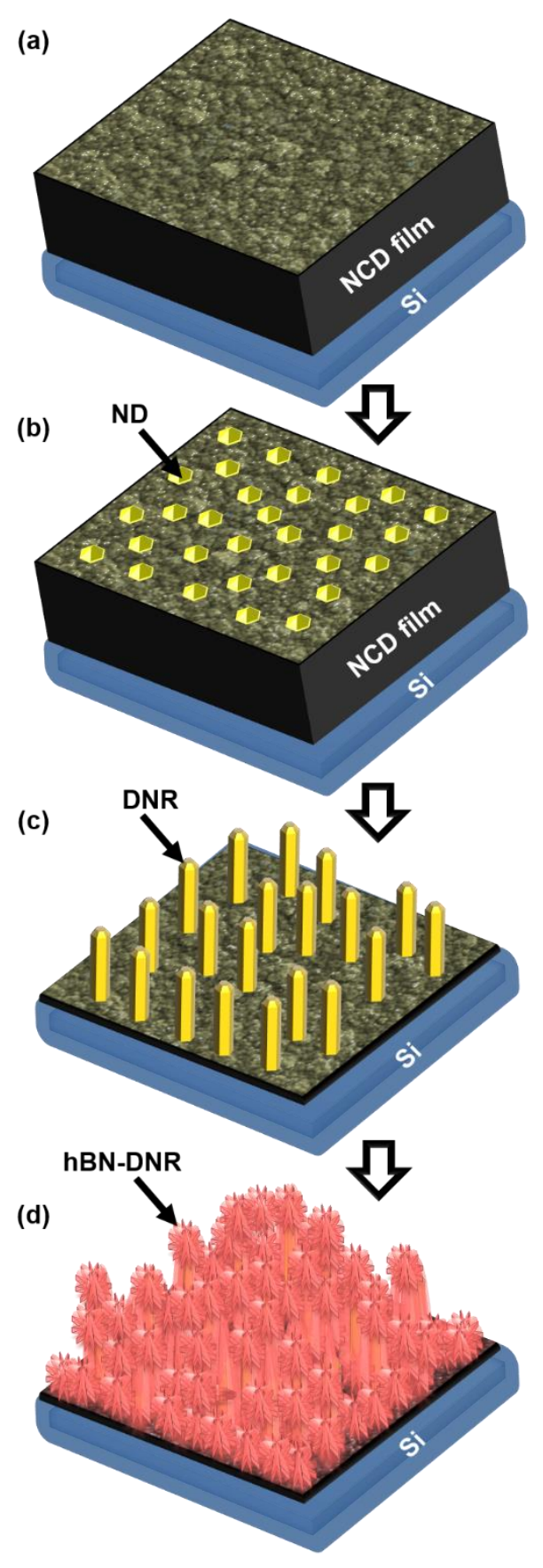

Fig. 1. 

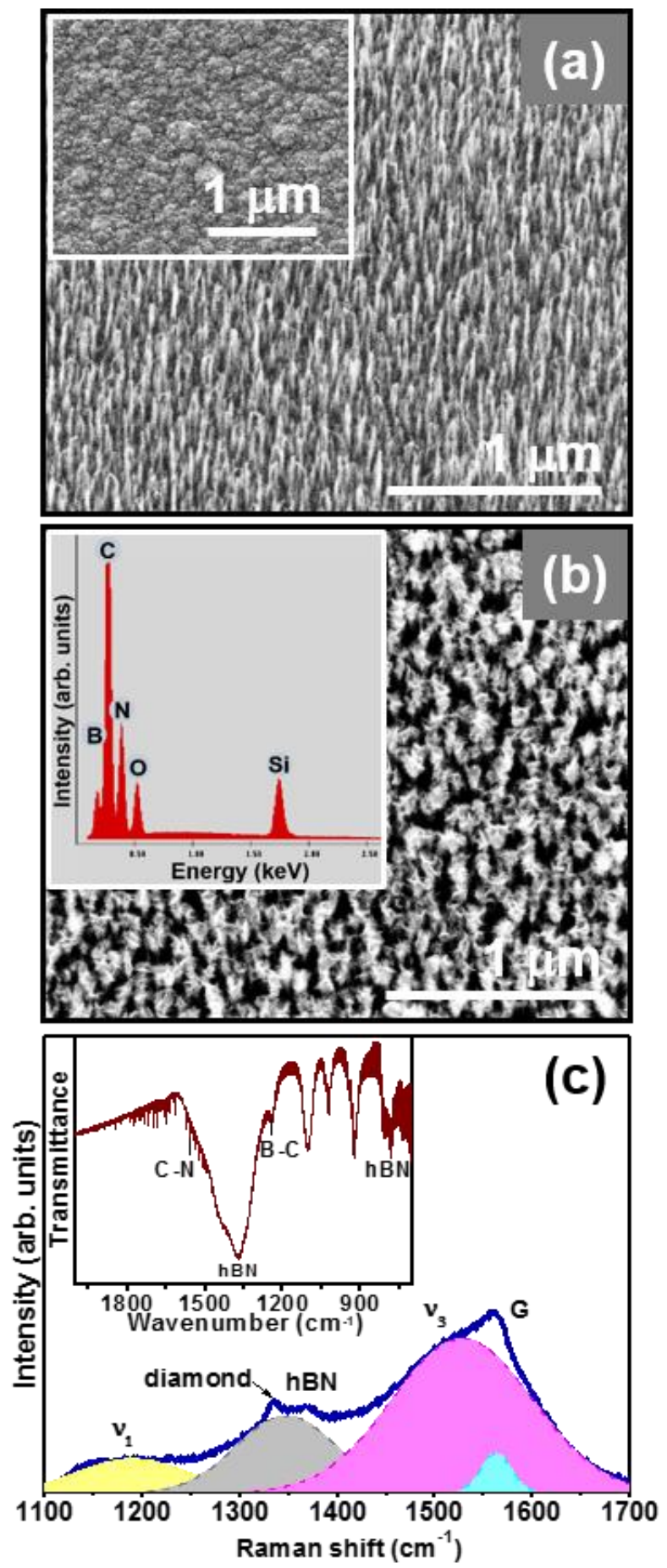

Fig. 2. 

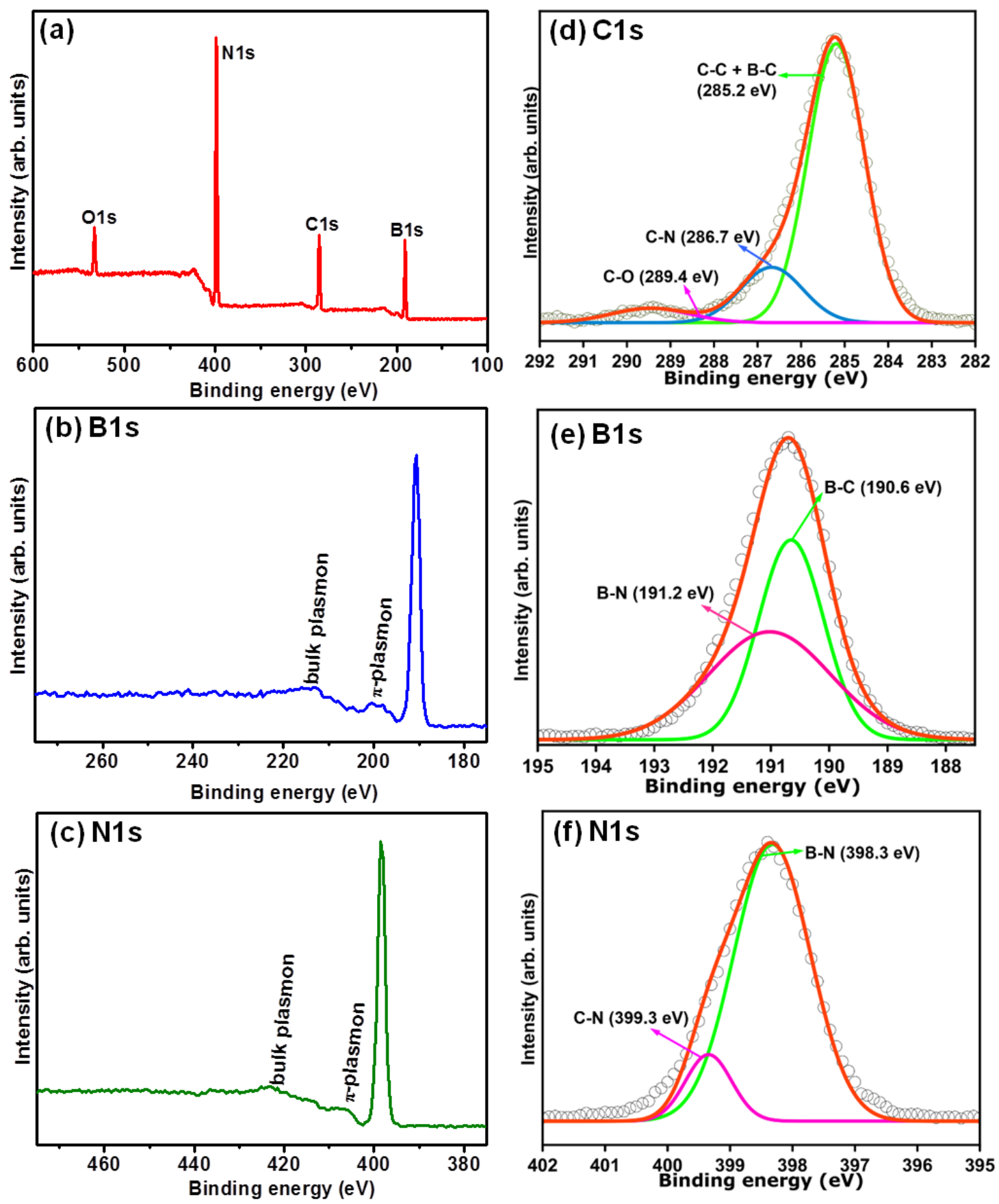

Fig. 3. 


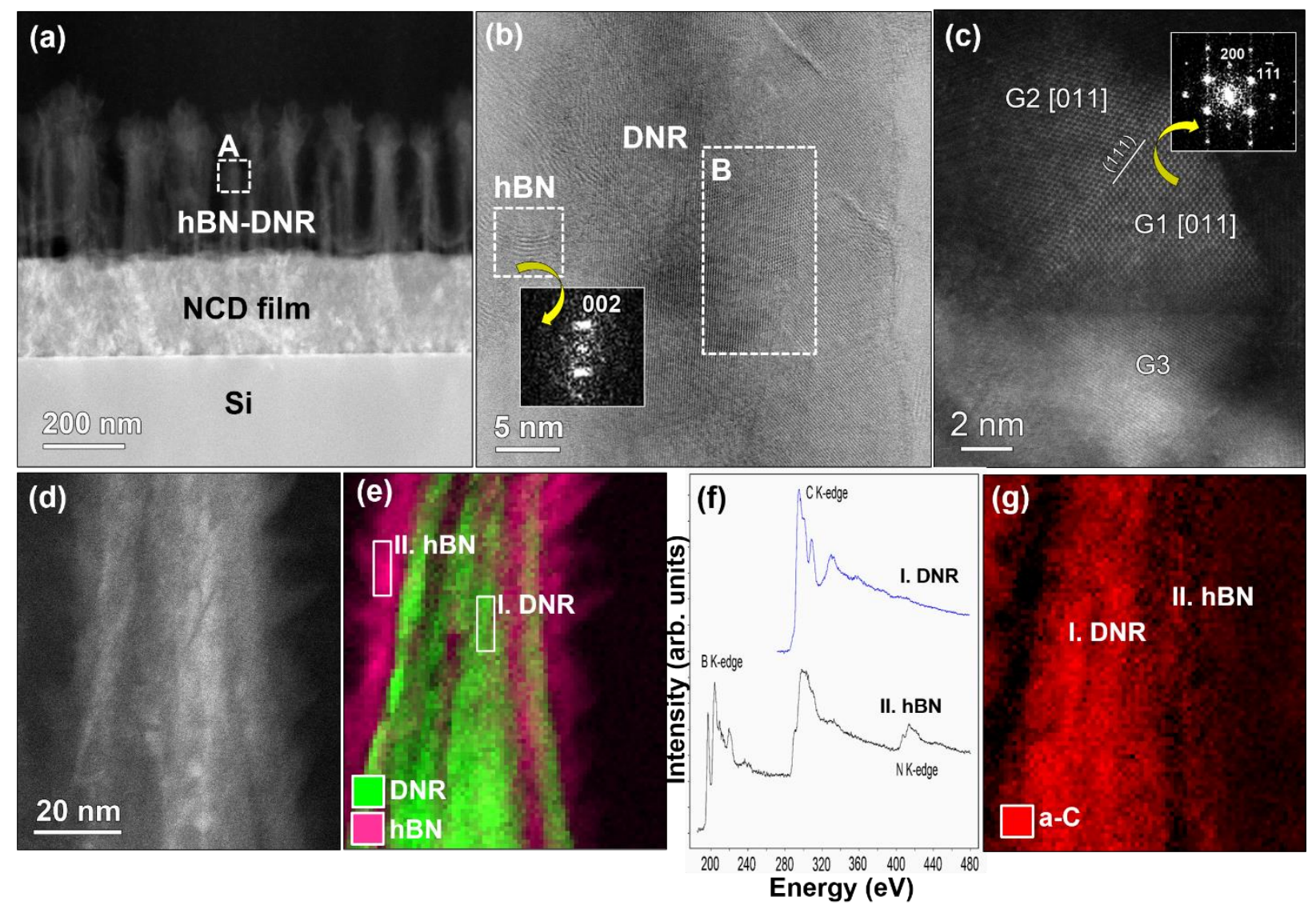

Fig. 4. 

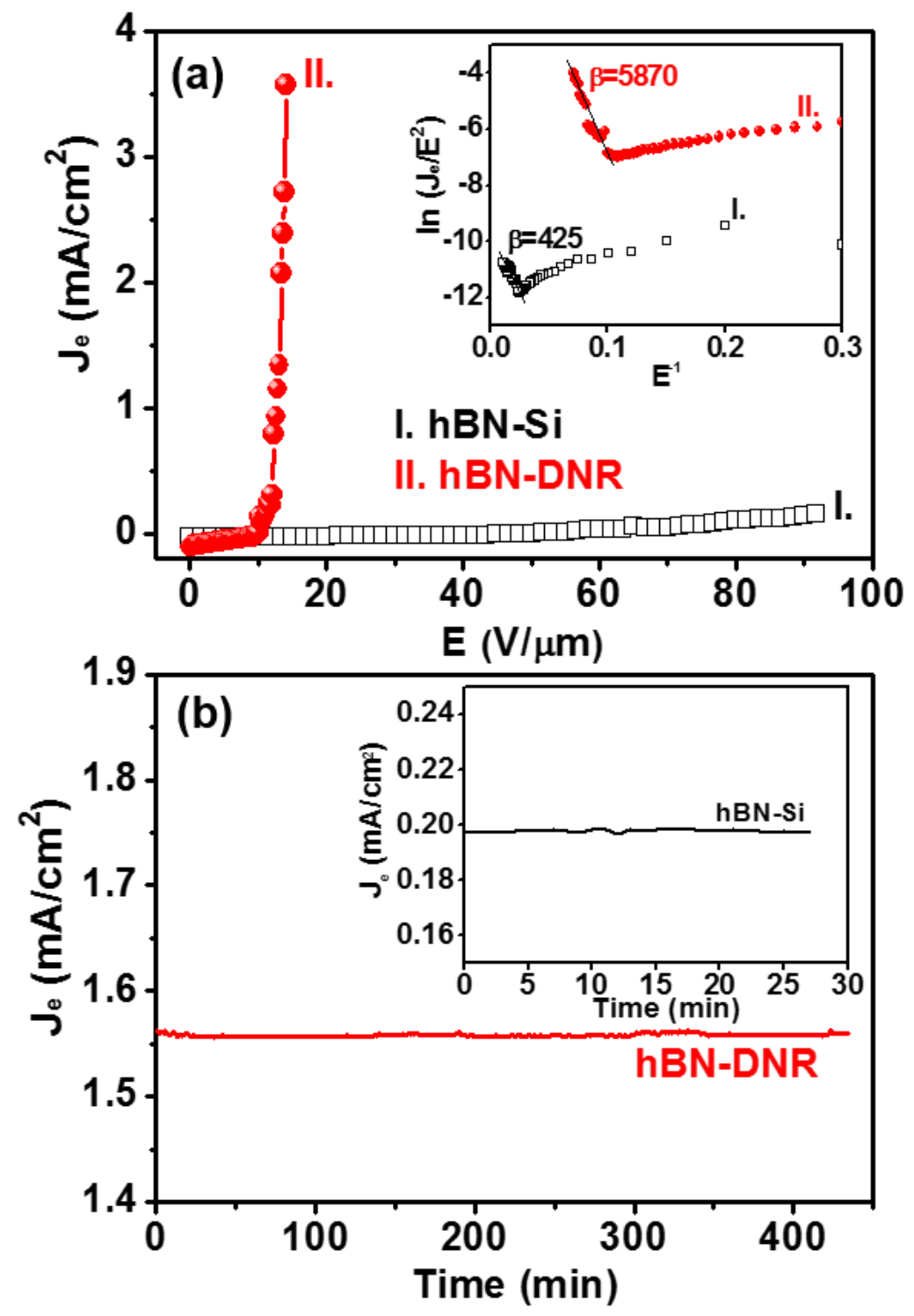

Fig. 5. 


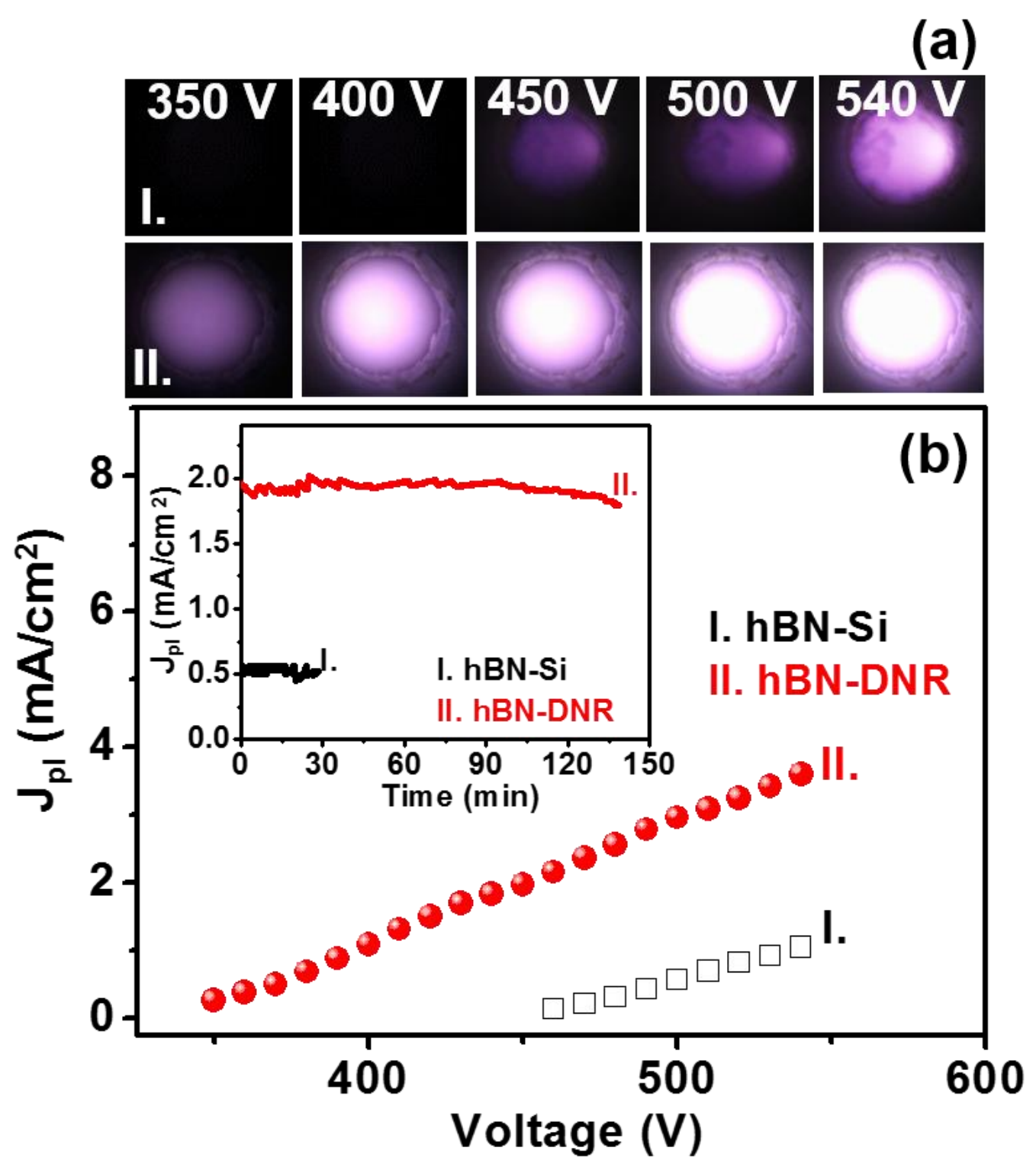

Fig. 6. 